\title{
The Role of Aberration-Corrected STEM in the Characterization of Oxide Cathode Materials
}

\author{
P.J. Phillips ${ }^{1}$, D.P. Abraham² ${ }^{2}$ J. Bareño ${ }^{2}$, C. Kim³ ${ }^{3}$, T. Yi ${ }^{3}$, J. Cabana ${ }^{3}$, R.F. Klie ${ }^{1}$ \\ ${ }^{1}$ Department of Physics, University of Illinois at Chicago, Chicago IL 60607 \\ ${ }^{2}$ Chemical Sciences \& Engineering, Argonne National Laboratory, Argonne, IL 60439 \\ ${ }^{3}$ Department of Chemistry, University of Illinois at Chicago, Chicago, IL 60607
}

The development of next-generation batteries for use in advanced applications (such as hybrid electric vehicles) continues to be a sought-after goal and thus a relevant research topic, in particular with respect to Li-ion and multivalent-ion batteries. In the case of Li-ion, their full potential has yet to be reached given their inherent cycling performance issues, such as voltage instability and capacity fading. On the other hand, by extending battery technology to include multivalent ions, it is proposed that such systems can surpass the energy storage capabilities of Li-ion technology, hence the impetus to identify materials which can accommodate (de)intercalation of such multivalents.

The present contribution will discuss a variety of oxide cathode materials in the context of scanning transmission electron microscopy (STEM) analysis. Specifically, these materials require characterization of chemical compositions/gradients, electronic structure (ion valence), structural disorder/rearrangements, and in the largely-unknown realm of multivalent ions: whether or not intercalation has actually occurred. Thus, STEM-based methods are quickly becoming the most promising characterization tools, with techniques available which include the direct imaging of both heavy and light elements, and both energy-dispersive X-ray (EDX) and electron energy loss (EEL) spectroscopies.

Two Li-based cathode materials will be discussed: a Mn-rich spinel and the monoclinic $\mathrm{Li}_{2} \mathrm{MnO}_{3}$. Nanocrystalline spinel particles were engineered with an Al-rich passivating epitaxial shell [1] to curb the common issue of degradation at the electrolyte/electrode interface; as such, the STEM will focus on directly imaging the shell in low angle annular dark field (LAADF) to confirm its epitaxial nature and composition analysis via EDX, as presented in Fig. 1. $\mathrm{Li}_{2} \mathrm{MnO}_{3}$ will be discussed in its pristine, electrochemically cycled, and in-situ electron beam irradiated states [2]; the latter allows for single particle tracking of the dynamic processes occurring upon $\mathrm{Li}$ and $\mathrm{O}$ loss from the material. In this case, the focus will be on tracking structural evolution (and hence the use of annular bright field (ABF) STEM) as well as the Mn valence and O loss via high-resolution EELS (Fig. 2).

Additionally, multivalent-based cathodes will be discussed. For these cases, generally a combinatorial approach is required, consisting of LAADF/ABF imaging, EELS to determine valence, and highspatial-resolution EDX spectroscopy to determine the nature of intercalation; furthermore, in some cases, the imaging voltage may change between $80 / 200 \mathrm{kV}$, depending on the desired output. It is important to note that much of the above analysis would not be possible without the use of advanced STEM instruments, outfitted with both EEL and EDX capabilities. In the present case, the UIC

aberration-corrected JEOL JEM-ARM 200CF STEM instrument, capable of $0.73 \AA$ spatial and 0.35 eV energy resolution, equipped with a large angle silicon drift EDX detector, was employed [3].

References

[1] C. Kim, P.J. Phillips, L. Xu, A. Dong, R. Buonsanti, R.F. Klie, J. Cabana, Chem. Mater. 27 (2015) 394-399. [2] P.J. Phillips, H. Iddir, D.P. Abraham, R.F. Klie, Appl. Phys. Lett. 105 (2014) 113905.

[3] Supported by an MRI-R ${ }^{2}$ grant from the National Science Foundation (Grant No. DMR-0959470).

[4] Support is acknowledged from the Joint Center for Energy Storage Research, an Energy Innovation Hub funded by the U.S. Department of Energy, Office of Science, Basic Energy Sciences. 

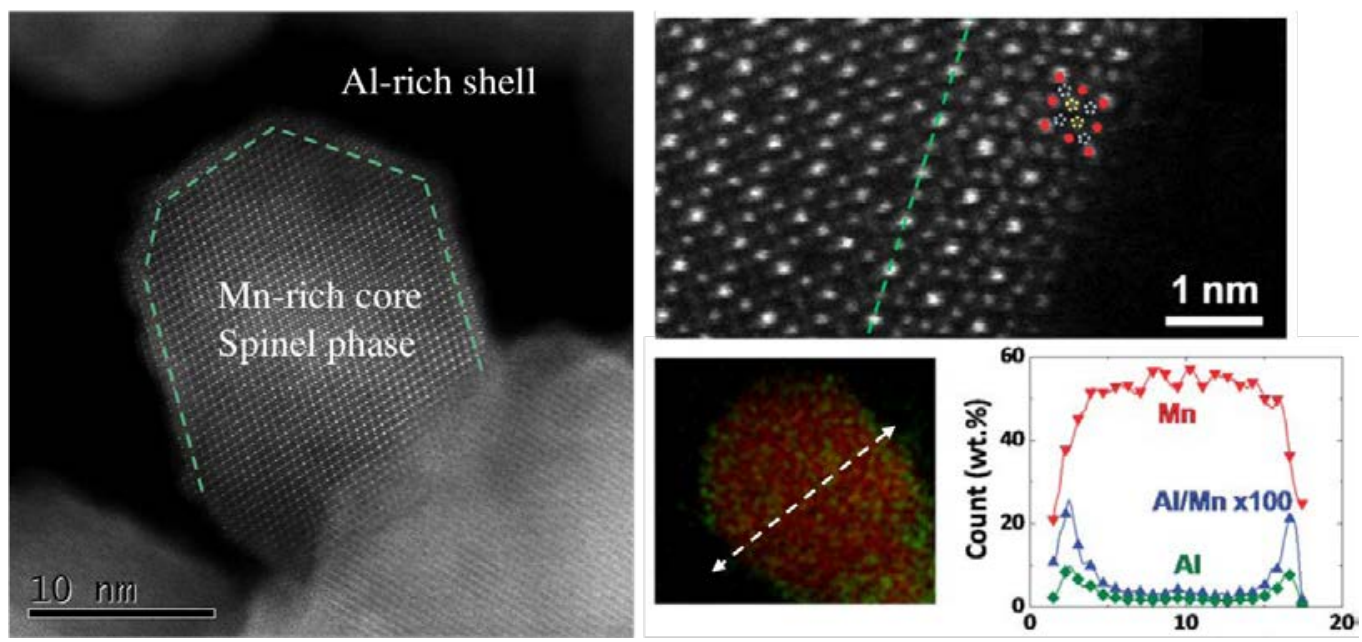

Figure 1: LAADF STEM image of the core-shell nanocrystals (left), along with a higher magnification view of the epitaxial shell (right); the Mn atomic positions are indicated with solid red spheres, while $\mathrm{O}$ and $\mathrm{Li}$ atomic positions are represented by hollow blue and yellow spheres, respectively, in the spinel structure. The Li site would not show intensity in this LAADF view, indicative that there are heavier elements present (Al). Finally, an EDX map and line scan of a core-shell nanoparticle, showing Al enrichment at the surface (shell).

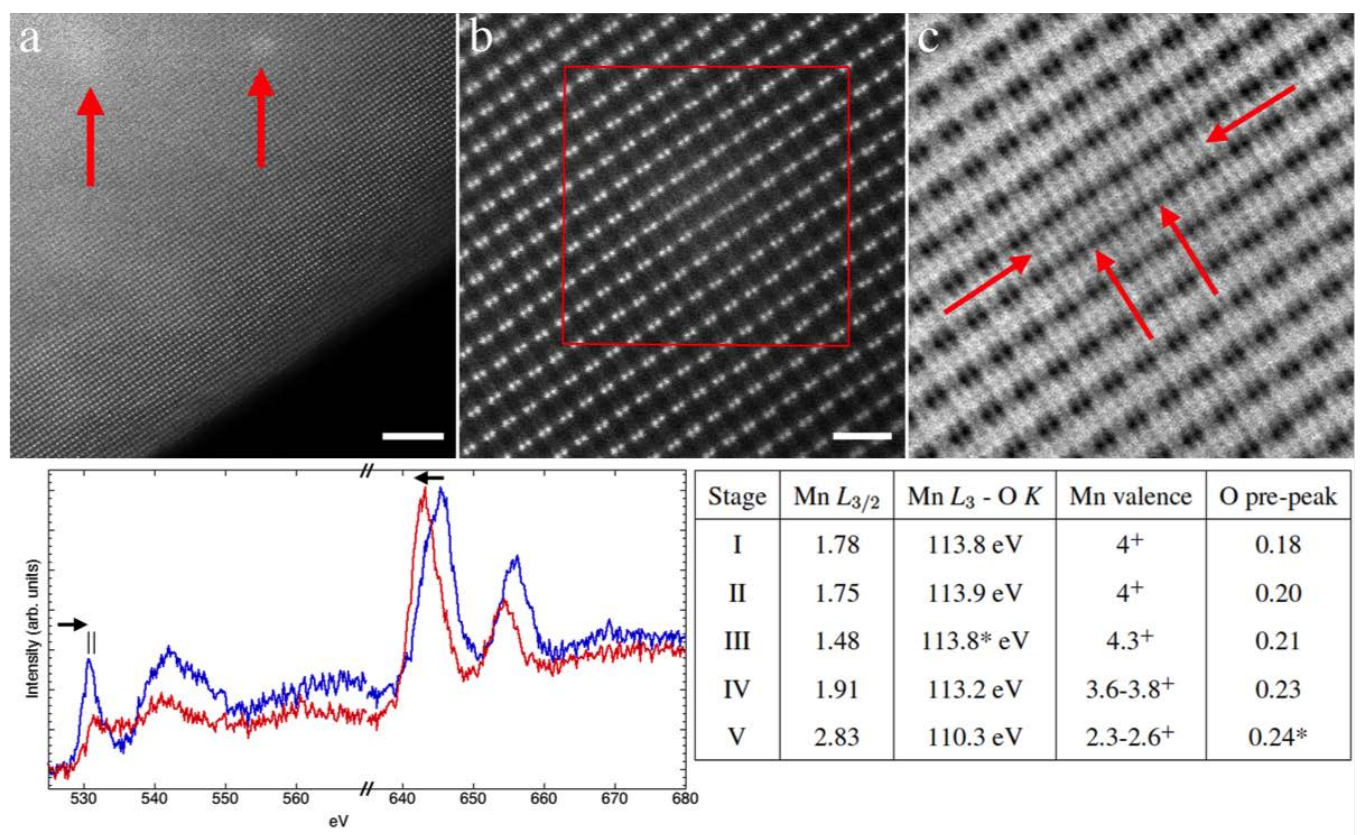

Figure 2: a) LAADF image showing two pockets of damage formed during electron beam irradiation; LAADF (b) and $\mathrm{ABF}$ (c) of a damaged region, showing anomalous intensity in what would be Li positions in the pristine oxide (arrowed); this is indicative of Mn moving into the Li sites. EELS O K- and Mn L-edge results form a moderately damaged (blue) and a severely damaged (red) region, with severely damaged material showing a decrease in the $\mathrm{Mn}$ valence and the presence of $\mathrm{O}$ vacancies; the table at right summarizes EELS results from various stages of damage, I being the pristine material, V being the most severely damaged (red EELS curve). 